\title{
Communication: High pressure specific heat spectroscopy reveals simple relaxation behavior of glass forming molecular liquid
}

\author{
Roed, Lisa Anita; Niss, Kristine; Jakobsen, Bo
}

Published in:

Journal of Chemical Physics

DOI:

$10.1063 / 1.4936867$

Publication date:

2015

\section{Document Version}

Publisher's PDF, also known as Version of record

Citation for published version (APA):

Roed, L. A., Niss, K., \& Jakobsen, B. (2015). Communication: High pressure specific heat spectroscopy reveals simple relaxation behavior of glass forming molecular liquid. Journal of Chemical Physics, 143, [221101]. https://doi.org/10.1063/1.4936867

\section{General rights}

Copyright and moral rights for the publications made accessible in the public portal are retained by the authors and/or other copyright owners and it is a condition of accessing publications that users recognise and abide by the legal requirements associated with these rights.

- Users may download and print one copy of any publication from the public portal for the purpose of private study or research.

- You may not further distribute the material or use it for any profit-making activity or commercial gain.

- You may freely distribute the URL identifying the publication in the public portal.

If you believe that this document breaches copyright please contact rucforsk@kb.dk providing details, and we will remove access to the work immediately and investigate your claim. 


\section{AlP $\mid \begin{aligned} & \text { The Journal of } \\ & \text { Chemical Physics }\end{aligned}$}

Communication: High pressure specific heat spectroscopy reveals simple relaxation behavior of glass forming molecular liquid

Lisa Anita Roed, Kristine Niss, and Bo Jakobsen

Citation: The Journal of Chemical Physics 143, 221101 (2015); doi: 10.1063/1.4936867

View online: $\mathrm{http}: / / d x$. doi.org/10.1063/1.4936867

View Table of Contents: http://scitation.aip.org/content/aip/journal/jcp/143/22?ver=pdfcov

Published by the AIP Publishing

\section{Articles you may be interested in}

Glass transition of ionic liquids under high pressure

J. Chem. Phys. 140, 244514 (2014); 10.1063/1.4885361

Dielectric spectroscopy and ultrasonic study of propylene carbonate under ultra-high pressures

J. Chem. Phys. 137, 084502 (2012); 10.1063/1.4746022

Dynamics of glass-forming liquids. XV. Dynamical features of molecular liquids that form ultra-stable glasses by vapor deposition

J. Chem. Phys. 135, 124515 (2011); 10.1063/1.3643332

The relative contributions of temperature and volume to structural relaxation of van der Waals molecular liquids

J. Chem. Phys. 118, 4578 (2003); 10.1063/1.1545449

Decoupling of the dc conductivity and ( $\alpha-)$ structural relaxation time in a fragile glass-forming liquid under high pressure

J. Chem. Phys. 116, 9882 (2002); 10.1063/1.1473819

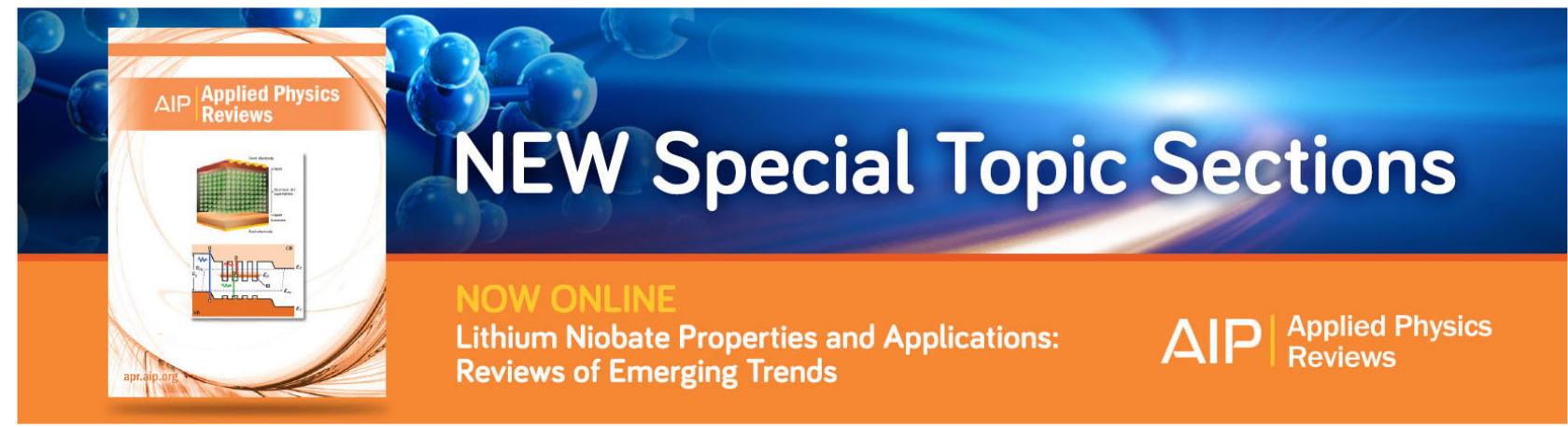




\title{
Communication: High pressure specific heat spectroscopy reveals simple relaxation behavior of glass forming molecular liquid
}

\author{
Lisa Anita Roed, Kristine Niss, and Bo Jakobsen ${ }^{\text {a) }}$ \\ DNRF Centre "Glass and Time," IMFUFA, Department of Sciences, Roskilde University, P.O. Box 260, \\ DK-4000 Roskilde, Denmark
}

(Received 22 October 2015; accepted 18 November 2015; published online 9 December 2015)

\begin{abstract}
The frequency dependent specific heat has been measured under pressure for the molecular glass forming liquid 5-polyphenyl-4-ether in the viscous regime close to the glass transition. The temperature and pressure dependences of the characteristic time scale associated with the specific heat is compared to the equivalent time scale from dielectric spectroscopy performed under identical conditions. It is shown that the ratio between the two time scales is independent of both temperature and pressure. This observation is non-trivial and demonstrates the existence of specially simple molecular liquids in which different physical relaxation processes are both as function of temperature and pressure/density governed by the same underlying "inner clock." Furthermore, the results are discussed in terms of the recent conjecture that van der Waals liquids, like the measured liquid, comply to the isomorph theory. (C) 2015 AIP Publishing LLC. [http://dx.doi.org/10.1063/1.4936867]
\end{abstract}

The viscosity of liquids close to the glass transition is strongly temperature dependent - just a few percent decrease in temperature can lead to several decades increase in viscosity. Coupled to the increase in viscosity is a slowing down of the molecular structural relaxation time (the alpha relaxation time), leading to a time scale separation between the fast iso-structural degrees of freedom and the slow structural degrees of freedom. The glass transition occurs at the temperature where the slow degrees of freedom are no longer accessible on the experimental time scale. This gives rise to a drop in the measured specific heat which is a classical signature of the glass transition. ${ }^{1}$ The time scale separation in the highly viscous liquid just above the glass transition temperature leads to time or equivalently frequency dependence of physical properties which couple to the structural relaxation; the mechanical moduli and the dielectric constant are, for example, frequency dependent. Even though heat capacity is a classical probe of the glass transition, the awareness of the fact that the specific heat is also a frequency dependent response function which shows relaxation is surprisingly young and only dates back to the 1980s (some of the earliest discussions are given in Refs. 2-7). The first experimental specific heat spectroscopic techniques for studying viscous liquids were also developed in the 1980s by Birge and Nagel ${ }^{4}$ and Christensen. ${ }^{5}$ The amount of data on frequency dependent specific heat is still very limited today 30 years later, probably because no standard commercial technique has been available. (The following list is not a comprehensive list of all specific heat spectroscopy studies of glass forming liquids but covers to the best of our knowledge all groups that have addressed the issue, Refs. 4, 5, and 8-14).

The workhorse in the study of frequency dependent response of glass forming liquids is dielectric spectroscopy

\footnotetext{
a)Electronic mail: boj@dirac.ruc.dk
}

both when it comes to studies of the temperature dependence of the alpha relaxation (e.g., Refs. 15-17) and the spectral shape of the relaxation (i.e., stretching ${ }^{18,19}$ and betarelaxation ${ }^{20}$ ). The use of dielectric spectroscopy is particularly dominant when it comes to high pressure studies, which have become increasingly important during the last couple of decades. Today, it is clear that the dynamics of viscous liquids should be understood as function of both temperature and pressure/density because this is the only way to disentangle the effect of density from that of thermal energy. Two key findings from high pressure studies are: density scaling (e.g., Refs. 21-24), and isochronal superposition (e.g., Refs. 25-30). These results are almost exclusively based on dielectric data because dielectric spectroscopy is easily adapted to high pressure and commercial equipment is available. ${ }^{31}$

Different physical properties probe the microscopic dynamics in different ways. An example is that dielectric spectroscopy only is sensitive to degrees of freedom which involve reorientation of dipoles in the liquid while specific heat measures those degrees of freedom which couple to changes in energy. This naturally leads to differences in the observed characteristic time scales for different properties it is, e.g., well-known that shear-mechanical relaxation is faster than dielectric relaxation (e.g., Ref. 32 and references therein).

While all the liquid dynamics slows down upon cooling (or compression) it is by no means trivial that all time scales follow each other as a function of temperature and pressure. A well-known example is the pronounced decoupling between translational and rotational motions which has been confirmed in many systems, also under pressure (e.g., Ref. 33). Even time scales which at a first coarse look seem to follow each other over many decades have been shown to have differences in the temperature dependence when analyzed in detail (e.g., Refs. 32 and 34). Moreover, there is an increasing amount of evidence for dynamical heterogenities in viscous liquids 
(e.g., Ref. 35). Different physical properties will a priori "see" and average differently over the dynamical heterogeneities. This would imply different temperature-dependence of the time scales as the dynamical heterogenities evolve with cooling and lead to the picture suggested by Angell in 1991, namely, a series of decoupling temperatures as the liquid is cooled down. ${ }^{36}$

An example of the pitfall of exclusively basing analysis on one response function is the understanding of the dynamics in monohydroxy alcohols. It was for a long time thought that the main dielectric relaxation peak was the signature of the structural relaxation, leading to puzzling observations about monohydroxy alcohols (e.g., in Ref. 18). This was only resolved when other response functions were analyzed, showing that the dominant process in the dielectric spectrum is not related to the structural molecular relaxation ${ }^{37}$ seen in specific heat ${ }^{38}$ and shear modulus. ${ }^{39,40}$

It is in itself a fundamental question whether the liquid relaxation seen in different techniques behaves in the same way. Additionally, it is important to establish whether dielectric results especially at elevated pressures can be generalized and viewed as generic information about the alpha relaxation.

Experimentalists have often attempted to find general (universal) behaviors and correlations in the effort to guide theory and models for the viscous slowing down. ${ }^{18,41-43}$ However, as more and more systems are studied, these results are usually found to hold only for a limited class of systems. ${ }^{44-48}$ Another trend is to focus on exotic phenomena seen in complicated systems like the notorious counter example water. ${ }^{49,50}$ The emerging picture is that while the viscous slowing down and the glass transition as such are universal features which (at least in principle) can be observed in all systems independent of chemical details, there is also a myriad of specific behaviors and it seems unlikely to capture all this in one simple model. Based on this understanding our proposal is to address the question of what the simplest behavior is? Or put in other words what are the features that should be included in the "ideal gas model" of glass forming liquids?

In this work, we present frequency dependent specific heat measurements taken as function of both temperature and pressure up to $300 \mathrm{MPa}$ on the molecular glass former 5-polyphenyl-4-ether (5PPE) and compare to existing dielectric data ${ }^{30,51}$ taken in the same pressure equipment ensuring consistency of absolute temperature and pressure. To the best of our knowledge, these are the first ever high pressure data where both dielectric and specific heat spectroscopy are taken under identical conditions, allowing for a detailed comparison.

5PPE has been studied intensively by the "Glass and time" group over the past years (at atmospheric pressure, ${ }^{14,32,52-55}$ and at high pressures ${ }^{30,56,57}$ ) and has been found to have a particular simple behavior. One of these findings was that no decoupling is seen in 5PPE when comparing the temperature dependence of 6 different time scales. ${ }^{54}$ This result was later supported by result from the group of Schick who compared dielectric and specific heat spectroscopy with lower accuracy but over larger range in dynamics. ${ }^{58}$
The data in the current paper extend these studies to high pressures. Hereby addressing the question of whether the alpha relaxation time is uniquely determined independent of probe in the entire phase space.

The strong temperature dependence of the characteristic time scales of liquids close to the glass transition has the consequence that even small temperature/pressure differences will lead to large errors in the measured time scales. In order to make detailed comparisons, it is therefore crucial to measure different response functions under the same conditions (see e.g., Ref. 59). To the best of our knowledge there is only one previous study of frequency dependent specific heat at elevated pressures, in which Leyser et al. ${ }^{60} 20$ years ago investigated orthoterphenyl in a limited pressure range up to $105 \mathrm{MPa}$, and compared to existing literature data. ${ }^{61}$ Their data show that the time scales do follow rather closely along the investigated paths in phase space; however, some systematic changes are seen. Due to the limited pressure/temperature range investigated and the fact that the dielectric data they compare to is from a different study, it is not possible to conclude if the ratio between the two time scales is constant or changing for this liquid.

Our unique specific heat spectroscopy technique is based on measuring the thermal impedance at a spherical surface in the liquid (the relation between heat flow into the liquid from the surface, and the temperature at the same surface). The method is inspired by the method developed by Birge and Nagel 30 years ago. ${ }^{4,62}$ Our version of the technique ${ }^{14}$ utilize a spherical geometry, and a liquid layer much thicker than the thermal wavelength (the thermally thick limit). In this case, the outer thermal and mechanical boundaries do not influence the measured property. ${ }^{63}$ What is measured is the longitudinal volume specific heat $c_{l}{ }^{63,64}$ which is approximately equal to the isobaric specific heat. ${ }^{65}$ By the 3omega technique a temperature dependent resistor is utilized as heat generator and thermometer simultaneous. ${ }^{14,62}$

The method is well suited to be adopted to different sample environments with little requirements to the mechanical properties of the sample cell and electrical connections. Altogether this makes the method perfect for integration in existing pressure equipment.

The measurements were performed using commercial high-pressure equipment from Unipress Equipment (Warsaw, Poland). The pressure is applied using a pressure liquid, which is separated from the sample cell by a shielding of rubber and Teflon. Pressures go up to $600 \mathrm{MPa}$ with a stability of $3 \mathrm{MPa}$, temperatures ranges from 233 to $333 \mathrm{~K}$ (for further details see Refs. 51, 56, and 66). A spherical NTC-thermistor bead (a temperature dependent resistor, with "Negative Temperature Coefficient") is used as heat generator and thermometer and is placed in the middle of the sample cell with a distance of $\approx 10 \mathrm{~mm}$ from the closest sides ensuring approximately thermally thick conditions down to the millihertz range. The 5PPE liquid studied is the diffusion pump oil 5-polyphenyl4-ether acquired from Santovac. The liquid was dried for one hour under vacuum before use.

The specific heat measurements are performed at different temperatures along the isobars; $0.1 \mathrm{MPa}$ (atmospheric pressure), $150 \mathrm{MPa}$, and $300 \mathrm{MPa}$. The initial data analysis 
was performed as in Ref. 14. Examples of the imaginary part of the frequency dependent longitudinal specific heat at different temperatures and pressures are shown in Fig. 1(a). The peak frequency of the imaginary part, $f_{c_{l}, \text { lp }}$ (the losspeak frequency), defines a directly experimental accessible characteristic time scale $\left(\tau_{c_{l}}=1 /\left(2 \pi f_{c_{l}, \mathrm{lp}}\right)\right)$ for the specific heat relaxation. Figure 1(b). shows the relaxation times for all measured temperatures and pressures. The extreme temperature and pressure dependences of the relaxation time are clearly seen in this representation. The shown error bars refer to limitations in precision of the specific heat technique. ${ }^{14}$ There is moreover approximately 0.1 decade uncertainty on the relaxation time due to the limited stability of the pressure of $3 \mathrm{MPa}$.

The comparable dielectric spectroscopy measurements were taken in relation to Refs. 30 and 51 utilizing the same pressure equipment. The dielectric measurements were performed along the isobars; $0.1 \mathrm{MPa}, 100 \mathrm{MPa}, 200 \mathrm{MPa}$, $300 \mathrm{MPa}$, and $400 \mathrm{MPa}$. The relaxation time is again defined from the loss-peak frequency $\left(\tau_{\epsilon}=1 /\left(2 \pi f_{\epsilon, \text { lp }}\right)\right)$. Figure 2 shows the relaxation time as a function of temperature at the different pressures for both the specific heat and for the dielectric measurements. As mentioned earlier the fact that the data are taken under the same thermal and pressure conditions gives a unique possibility for directly comparing
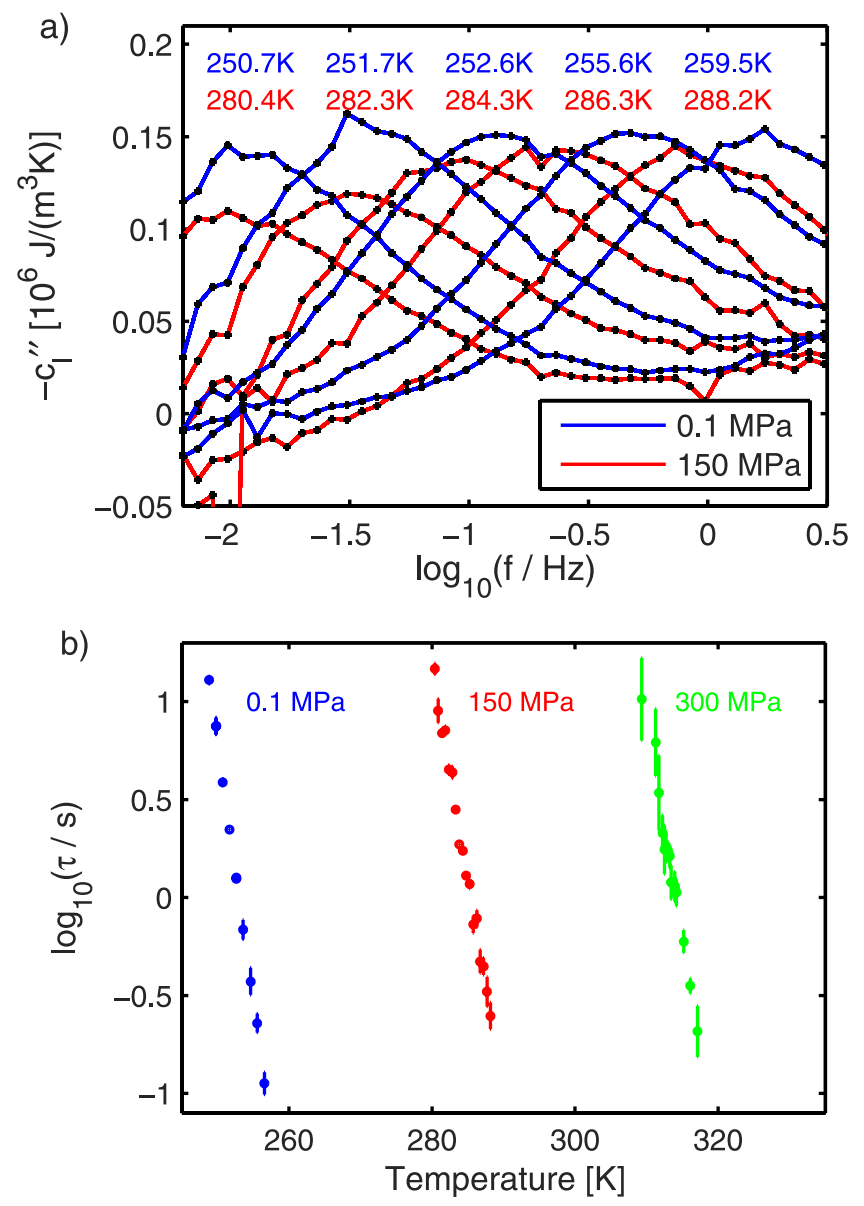

FIG. 1. Specific heat data. (a) The imaginary part of the longitudinal specific heat at the indicated temperatures and pressures. (b) The relaxation time $\tau$, based on the loss-peak frequency, as a function of temperature for all studied temperatures and pressures.

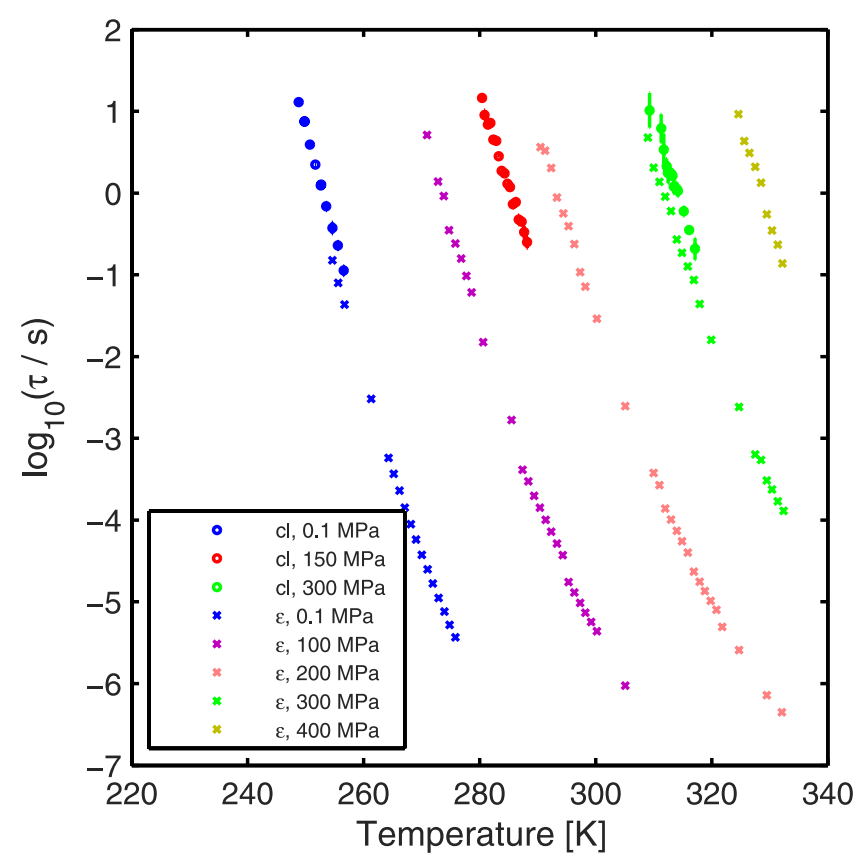

FIG. 2. Comparison of the relaxation times $(\tau)$ for the specific heat measurements (same data as on Fig. 1) and the dielectric measurements at the indicated pressures.

the temperature and pressure dependence of the time scale from dielectric and specific heat spectroscopy over a rather wide pressure range.

From Fig. 2, it is seen that the temperature dependence of the relaxation times from the two methods closely follows each other at all investigated pressures. It is also seen that the dielectric relaxation time is faster than the specific heat relaxation time at all pressures. This shows that the ambient pressure observation of identical temperature dependence of dielectric and specific heat relaxation time presented by some of us in Jakobsen et al. (2012) $)^{54}$ on 5PPE also holds at elevated pressures.

Figure 3 presents the same data using isochrones, which are lines in the phase diagram of constant relaxation time; that is contour lines of the relaxation time map, $\tau(T, P)$. The isochrones are illustrated for both methods, and it is seen that the isochrones for the two response functions are parallel.

The main result of this communication is that the $\tau_{\epsilon} / \tau_{c_{l}}$ ratio is constant within error bars for all investigated temperatures and pressures for 5PPE. This result is obtained by combining the observation from Fig. 2 (and Ref. 54) and Fig. 3 as described below. Combining the data from the present study and from Ref. 54 yields $\log _{10}\left(\tau_{\epsilon} / \tau_{c_{l}}\right)=-0.4 \pm 0.1$, with the major contribution to the uncertainty coming from pressure instabilities.

The relaxation time is a smooth function of temperature and pressure, and parallel isochrones therefore imply that an isochrone for one of the two response functions is also an isochrone for the other but with a different time scale, as the dielectric relaxation is faster than the specific heat relaxation at a given state point.

The difference between the time scales associated with the isochrones is not a priory the same for different isochrones. The observation that the $\tau_{\epsilon} / \tau_{c_{l}}$ ratio is constant along the 


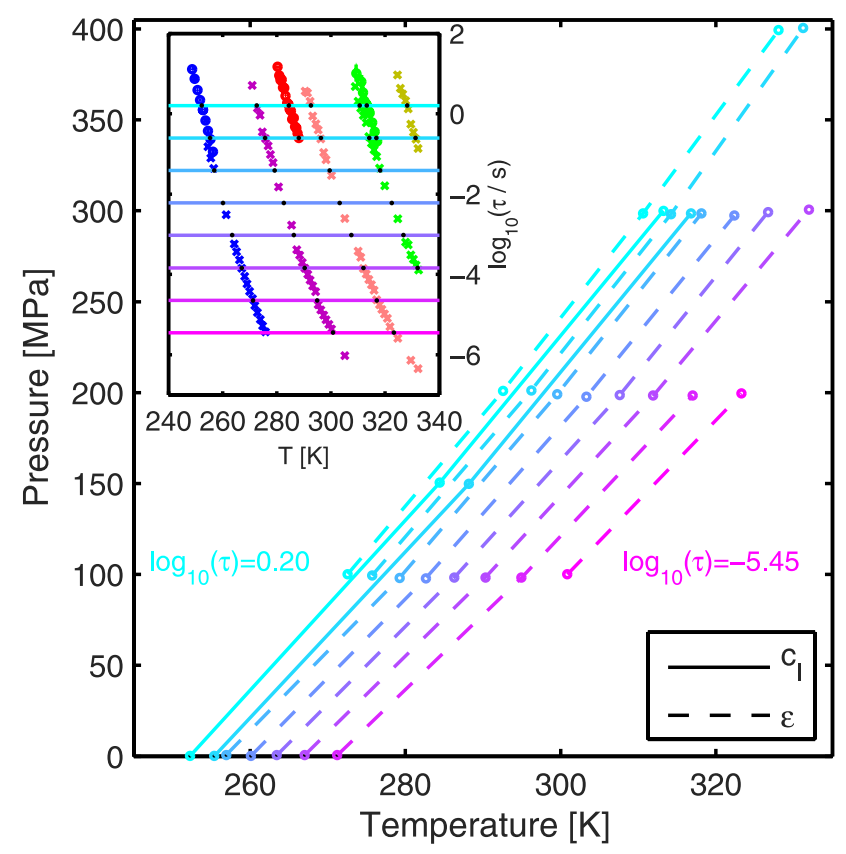

FIG. 3. Isochrones for specific heat (full lines) and dielectric (dashed lines). The two slowest isochrones are found for both the specific heat and dielectric, but the six fastest isochrones are only found for the dielectric measurements. The inset shows the same data as Fig. 2 where the vertical lines illustrate eight chosen relaxation times. The temperatures used for the isochrones are found by interpolation (indicated by small solid points on the inset).

isobars as shown in Fig. 2 (and illustrated even more clearly at atmospheric pressure in Ref. 54) is therefore an additional simplicity. Since any isobar crosses all the isochrones, we can conclude that the time scale difference associated with the isochrones indeed is the same in all of the explored part of the phase diagram for this liquid (it is enough to shown that it is constant along one curve, crossing the isochrones).

The observation that the isochrones from one of the methods are also isochrones for the other method is a direct prediction of the isomorph theory developed in the "Glass and Time" group. ${ }^{67-69}$ The theory predicts the existence of isomorphs which are curves in the phase diagram of simple (called Roskilde simple) liquids along which a number of properties are invariant. The isochrones of any response function will in the viscous regime (or in reduced units) coincide with an isomorphs, which means that an isochrone from one response function is also an isochrone for another response function, as all dynamics are invariant along an isomorph. ${ }^{30}$

Simple van der Waals liquids as 5PPE are expected to be Roskilde simple. ${ }^{67,68,70}$ We have in Refs. 30, 56, and 57 shown that 5PPE corroborates other isomorph predictions, this together with the present result indicating that 5PPE indeed is a good example of a Roskilde simple liquid.

In Jakobsen et al. (2012) ${ }^{54}$ some of us showed that at ambient pressure, not only specific heat and dielectric but also several other thermo-viscoelastic responses have time scales which follow closely with temperature for 5PPE. Based on this, we hypothesize that the time scales of all the frequency dependent response functions of 5PPE have a common temperature and pressure dependence in the viscous regime. An interpretation of this observation is that the structural relaxation time is governed by one "inner clock" and implies a greater simplicity than predicted by the isomorph theory. It is also a challenge to understand how all response functions can couple so closely if the underlying dynamics is heterogeneous with a growing length scale along with viscous slowing down - since the different macroscopic responses could very well average differently over the heterogeneities.

5PPE has other simple behaviors which are beyond isomorph theory. The spectral shape of the dielectric signal obeys Time Temperature Pressure Superposition (TTPS), thus it is independent of pressure and temperature in a range where $\tau_{\epsilon}$ changes by 7 orders of magnitude. ${ }^{30,51}$ Time temperature superposition is moreover found in frequency dependent bulk modulus and the shear mechanical relaxation, and these two moduli in fact have the same spectral shape. ${ }^{55}$ Finally 5PPE is also found to obey time aging time superposition. ${ }^{53}$

The simple behavior of 5PPE is obviously not universal for all glass-forming liquids, it might not even be a general behavior of van der Waals bonded liquids. Yet 5PPE exhibits the hallmarks of glass forming liquids; it has a non-Arrhenius temperature dependence (fragility index $\mathrm{m}$ $=80-85)^{71}$ and a non-exponential relaxation (high frequency power law -0.5). ${ }^{32}$ This means that models and theories for understanding non-Arrhenius non-exponential relaxation need to be consistent with a simple behavior where there is no decoupling of different time scales and no increase in the broadening of the relaxations in the entire viscous range (defined as time scales ranging from a microsecond up to a kilosecond).

To summarize, we have presented specific heat spectroscopy data over an unprecedented pressure range, with accompanying dielectric data taken under the same thermal and pressure conditions. The main experimental results are that the characteristic time scale of the specific heat and the dielectric relaxation follow each other closely as function of temperature at all investigated pressures and that isochrone curves for specific heat and dielectric spectroscopy coincide. The consequence of these two observations is that the $\tau_{\epsilon} / \tau_{c_{l}}$ ratio is constant over the investigated temperature and pressure ranges, with the dielectric spectroscopy being the fastest.

Altogether 5PPE seems to have the simplest possible behavior in respect to differences in time scales between response functions, namely, that one common inner clock controls the different relaxations, and the only difference is a temperature and pressure independent ratio between the different time scales.

L.A.R. and K.N. wish to acknowledge The Danish Council for Independent Research for supporting this work. The center for viscous liquid dynamics "Glass and Time" is sponsored by the Danish National Research Foundation's Grant No. DNRF61.

${ }^{1}$ W. Kauzmann, Chem. Rev. 43, 219 (1948).

${ }^{2}$ H. Gobrecht, K. Hamann, and G. Willers, J. Phys. E: Sci. Instrum. 4, 21 (1971).

${ }^{3}$ C. A. Angell and L. M. Torell, J. Chem. Phys. 78, 937 (1983).

${ }^{4}$ N. O. Birge and S. R. Nagel, Phys. Rev. Lett. 54, 2674 (1985).

${ }^{5}$ T. Christensen, J. Phys. Colloq. 46, C8-635 (1985).

${ }^{6}$ N. O. Birge, Phys. Rev. B 34, 1631 (1986).

${ }^{7}$ J. K. Nielsen and J. C. Dyre, Phys. Rev. B 54, 15754 (1996). 
${ }^{8}$ M. Settles, F. Post, D. Muller, A. Schulte, and W. Doster, Biophys. Chem. 43, 107 (1992).

${ }^{9}$ I. K. Moon, Y. H. Jeong, and S. I. Kwun, Rev. Sci. Instrum. 67, 29 (1996).

${ }^{10}$ T. Christensen and N. B. Olsen, J. Non-Cryst. Solids 235-237, 296 (1998)

${ }^{11}$ L. Carpentier, O. Bustin, and M. Descamps, J. Phys. D: Appl. Phys. 35, 402 (2002).

${ }^{12}$ E. H. Bentefour, C. Glorieux, M. Chirtoc, and J. Thoen, J. Appl. Phys. 93, 9610 (2003).

${ }^{13}$ A. A. Minakov, S. A. Adamovsky, and C. Schick, Thermochim. Acta 403, 89 (2003).

${ }^{14}$ B. Jakobsen, N. B. Olsen, and T. Christensen, Phys. Rev. E 81, 061505 (2010).

${ }^{15}$ R. Richert and C. A. Angell, J. Chem. Phys. 108, 9016 (1998).

${ }^{16}$ T. Hecksher, A. I. Nielsen, N. B. Olsen, and J. C. Dyre, Nat. Phys. 4, 737 (2008).

${ }^{17}$ Y. S. Elmatad, D. Chandler, and J. P. Garrahan, J. Phys. Chem. B 113, 5563 (2009).

${ }^{18}$ R. Bohmer, K. L. Ngai, C. A. Angell, and D. J. Plazek, J. Chem. Phys. 99, 4201 (1993).

${ }^{19}$ A. I. Nielsen, T. Christensen, B. Jakobsen, K. Niss, N. B. Olsen, R. Richert, and J. C. Dyre, J. Chem. Phys. 130, 154508 (2009).

${ }^{20}$ G. P. Johari and M. Goldstein, J. Chem. Phys. 53, 2372 (1970).

${ }^{21}$ C. Dreyfus, A. Aouadi, J. Gapinski, M. Matos-Lopes, W. Steffen, A. Patkowski, and R. M. Pick, Phys. Rev. E 68, 011204 (2003).

${ }^{22} \mathrm{C}$. Alba-Simionesco, A. Cailliaux, A. Alegría, and G. Tarjus, Europhys. Lett. 68, 58 (2004).

${ }^{23}$ R. Casalini and C. M. Roland, Phys. Rev. E 69, 062501 (2004).

${ }^{24}$ C. M. Roland, S. Hensel-Bielowka, M. Paluch, and R. Casalini, Rep. Prog. Phys. 69, 1405 (2005).

${ }^{25}$ A. Tölle, Rep. Prog. Phys. 64, 1473 (2001).

${ }^{26}$ C. Roland, R. Casalini, and M. Paluch, Chem. Phys. Lett. 367, 259 (2003).

${ }^{27}$ S. Pawlus, M. Paluch, M. Sekula, K. L. Ngai, S. J. Rzoska, and J. Ziolo, Phys. Rev. E 68, 021503 (2003).

${ }^{28}$ K. L. Ngai, R. Casalini, S. Capaccioli, M. Paluch, and C. M. Roland, J. Phys. Chem. B 109, 17356 (2005).

${ }^{29}$ C. M. Roland, R. Casalini, R. Bergman, and J. Mattsson, Phys. Rev. B 77, 012201 (2008).

${ }^{30}$ L. A. Roed, D. Gundermann, J. C. Dyre, and K. Niss, J. Chem. Phys. 139, 101101 (2013).

${ }^{31}$ Examples of commercial high pressure dielectric equipment are a full dielectric high pressure system from "NOVOCONTROL Technologies GmbH" and high pressure vessels with electrical feed throughs from "Unipress Equipment."

${ }^{32}$ B. Jakobsen, K. Niss, and N. B. Olsen, J. Chem. Phys. 123, 234511 (2005).

${ }^{33}$ S. H. Bielowka, T. Psurek, J. Ziolo, and M. Paluch, Phys. Rev. E 63, 062301 (2001).

${ }^{34}$ F. Stickel, E. W. Fischer, and R. Richert, J. Chem. Phys. 104, 2043 (1996).

${ }^{35}$ M. D. Ediger, Annu. Rev. Phys. Chem. 51, 99 (2000).

${ }^{36}$ C. A. Angell, J. Non-Cryst. Solids 131-133, 13 (1991).

${ }^{37}$ R. Böhmer, C. Gainaru, and R. Richert, Phys. Rep. 545, 125 (2014).

${ }^{38}$ H. Huth, L.-M. Wang, C. Schick, and R. Richert, J. Chem. Phys. 126, 104503 (2007).

${ }^{39}$ B. Jakobsen, C. Maggi, T. Christensen, and J. C. Dyre, J. Chem. Phys. 129, 184502 (2008).

${ }^{40}$ T. Hecksher and B. Jakobsen, J. Chem. Phys. 141, 101104 (2014).
${ }^{41}$ A. P. Sokolov, E. Rössler, A. Kisliuk, and D. Quitmann, Phys. Rev. Lett. 71, 2062 (1993).

${ }^{42}$ T. Scopigno, G. Ruocco, F. Sette, and G. Monaco, Science 302, 849 (2003).

${ }^{43}$ V. N. Novikov and A. P. Sokolov, Nature 431, 961 (2004).

${ }^{44}$ S. N. Yannopoulos and G. N. Papatheodorou, Phys. Rev. B 62, 3728 (2000).

${ }^{45}$ D. H. Huang and G. B. McKenna, J. Chem. Phys. 114, 5621 (2001).

${ }^{46}$ U. Buchenau and A. Wischnewski, Phys. Rev. B 70, 092201 (2004).

${ }^{47}$ K. Niss, C. Dalle-Ferrier, G. Tarjus, and C. Alba-Simionesco, J. Phys.: Condens. Matter 19, 076102 (2007).

${ }^{48}$ C. Dalle-Ferrier, K. Niss, A. P. Sokolov, B. Frick, J. Serrano, and C. Alba-Simionesco, Macromolecules 43, 8977 (2010).

${ }^{49}$ P. G. Debenedetti, J. Phys.: Condens. Matter 15, R1669 (2003).

${ }^{50}$ C. A. Angell, Science 319, 582 (2008).

${ }^{51}$ L. A. Roed, "Isochronal superposition - an experimental test of a prediction from the isomorph theory," Master's thesis, NSM, IMFUFA, Physics, Roskilde University, Denmark, 2012.

${ }^{52}$ K. Niss, B. Jakobsen, and N. B. Olsen, J. Chem. Phys. 123, 234510 (2005).

${ }^{53}$ T. Hecksher, N. B. Olsen, K. Niss, and J. C. Dyre, J. Chem. Phys. 133, 174514 (2010).

${ }^{54}$ B. Jakobsen, T. Hecksher, T. Christensen, N. B. Olsen, J. C. Dyre, and K. Niss, J. Chem. Phys. 136, 081102 (2012).

${ }^{55}$ T. Hecksher, N. B. Olsen, K. A. Nelson, J. C. Dyre, and T. Christensen, J. Chem. Phys. 138, 12A543 (2013).

${ }^{56} \mathrm{D}$. Gundermann, "Testing predictions of the isomorph theory by experiment," Ph.D. thesis, Roskilde University, DNRF Centre "Glass \& Time", IMFUFA, NSM, 2013).

${ }^{57}$ W. Xiao, J. Tofteskov, T. V. Christensen, J. C. Dyre, and K. Niss, J. NonCryst. Solids 407, 190 (2015).

${ }^{58}$ E. Shoifet, G. Schulz, and C. Schick, Thermochim. Acta 603, 227 (2015).

${ }^{59}$ R. Richert, Chem. Phys. Lett. 199, 355 (1992).

${ }^{60}$ H. Leyser, A. Schulte, W. Doster, and W. Petry, Phys. Rev. E 51, 5899 (1995).

${ }^{61}$ M. Naoki, H. Endou, and K. Matsumoto, J. Phys. Chem. 91, 4169 (1987).

${ }^{62}$ N. O. Birge, P. K. Dixon, and N. Menon, Thermochim. Acta 304-305, 51 (1997).

${ }^{63}$ T. Christensen and J. C. Dyre, Phys. Rev. E 78, 021501 (2008).

${ }^{64}$ T. Christensen, N. B. Olsen, and J. C. Dyre, Phys. Rev. E 75, 041502 (2007).

${ }^{65}$ The longitudinal volume specific heat $c_{l}$ is related to the isochoric specific

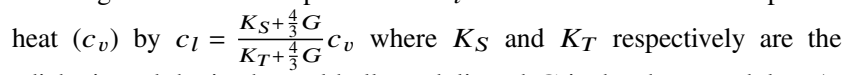
adiabatic and the isothermal bulk moduli, and $G$ is the shear modulus. At state points where relaxation occurs $G$ will differ from 0 , which means that $c_{l}$ and $c_{p}$ differs. However, the difference in characteristic timescale is very small (see supplementary material of Ref. 54).

${ }^{66}$ B. Igarashi, T. Christensen, E. H. Larsen, N. B. Olsen, I. H. Pedersen, T. Rasmussen, and J. C. Dyre, Rev. Sci. Instrum. 79, 045106 (2008).

${ }^{67}$ N. Gnan, T. B. Schrøder, U. R. Pedersen, N. P. Bailey, and J. C. Dyre, J. Chem. Phys. 131, 234504 (2009).

${ }^{68}$ J. C. Dyre, J. Phys. Chem. B 118, 10007 (2014).

${ }^{69}$ T. B. Schrøder and J. C. Dyre, J. Chem. Phys. 141, 204502 (2014).

${ }^{70}$ T. S. Ingebrigtsen, T. B. Schrøder, and J. C. Dyre, Phys. Rev. X 2, 011011 (2012).

${ }^{71} \mathrm{~K}$. Niss and B. Jakobsen, "Dielectric and shear mechanical relaxation in glass forming liquids - a thorough analysis and experimental test of the DiMarzio-Bishop model," Master thesis, Roskilde University (2003), text nr. 424 in "Tekster fra IMFUFA." 